${ }^{1}$ Московский клинический научнопрактический иентр им. А.С. Логинова

${ }^{2}$ Тверской государственный медицинский университет

${ }^{3}$ Московский государственный медико-

стоматологический университет им. А.И. Евдокимова

${ }^{4}$ Омский государственный медицинский университет

${ }^{5}$ Новосибирский государственный медииинский университет

${ }^{6}$ Кемеровская клиническая районная больнииа
Приверженность врачей первичного звена рекомендациям по диагностике и лечению заболеваний, ассоциированных с Helicobacter pylori Д.С. Бордин, д.м.н., проф. ${ }^{1-3}$, Т.С. Кролевец, к.м.н. ${ }^{4}$ М.А. Ливзан, д.м.н., проф.. ${ }^{4}$ М.Ф. Осипенко, д.м.н., проф.. ${ }^{5}$ Л.И. Токарева ${ }^{6}$, Е.В. Чебаненко ${ }^{4}$

Адрес для переписки: Татьяна Сергеевна Кролевец, mts-8-90@mail.ru

Для цитирования: Бордин Д.С., Кролевеи Т.С., Ливзан М.А. и др. Приверженность врачей первичного звена рекомендациям по диагностике и лечению заболеваний, ассоциированных с Helicobacter pylori // Эффективная фармакотерапия. 2019. Т. 15. № 36. С. $22-31$.

DOI 10.33978/2307-3586-2019-15-36-22-31

Статья подготовлена на базе Федерального государственного бюджетного образовательного учреждения высшего образования «Омский государственный медицинский университет» Министерства здравоохранения Российской Федерации.

Цель - оценить приверженность специалистов первичного звена выполнению рекомендаций по диагностике и лечению заболеваний, ассочиированных с Helicobacter pylori.

Материал и методы. Проведено анонимное анкетирование 221 врача (154 (69,7\%) терапевта и 67 (30,3\%) гастроэнтерологов), работающего в лечебно-профилактических учреждениях пяти городов России. Уровень согласия респонденты выражали в баллах: 0 - не знаю, 1 - не согласен, 2 - согласен не полностью и 3 - полностью согласен.

Результать. Врачи были полностью согласны диагностировать и лечить Н. pуlогі-инфекиию при язвенной болезни в период обострения (90,5\%), у родственников первой линии родства больных раком желудка (81,4\%), при хроническом атрофическом гастрите (77,8\%). Врачи выразили согласие с выполнением эрадикации при планировании длительного приема ингибиторов протонной помпь - 38,9\% случаев, нестероидных противовоспалительных препаратов - 42,6\%, у больных с симптомами диспепсии - 41,2\% случаев. Достоверно значимо чаще несогласие регистрировалось среди терапевтов со стажем до 20 лет, не имевших категории ( $p \leq 0,05)$. Респонденты предпочли выполнение гистобактериоскопии как для первичной диагностики, так и для контроля после лечения (76,9 и 64,7\%). В пользу серологических методов проголосовали 43,9 и 14,5\% врачей соответственно. Специиалисты были готовы использовать дыхательный тест и исследование кала как в иелях первичной диагностики (56,3 и 55,4\%), так и после лечения (58,4 и 55,7\%). В отночении схем лечения врачи продемонстрировали приверженность стандартной тройной терапии с кларитромииином в качестве первой тинии (80,9\%), показатель достоверно выше среди терапевтов ( $\chi 2=18,2540 ; p \leq 0,05)$. Респонденты в большей степени обеспокоены низкой приверженностью лечению пациентов (93,4\%) и ограниченностью в диагностических возможностях (85,3\%).

Заключение. Выявлено несогласие врачей с рядом ключевых рекомендачий экспертов, что негативно отражается на качестве диагностики и эбфективности лечения заболеваний, ассоциированных с H. pylori.

Ключевые слова: согласие, диагностика и лечение Helicobacter pylori, рекомендации 


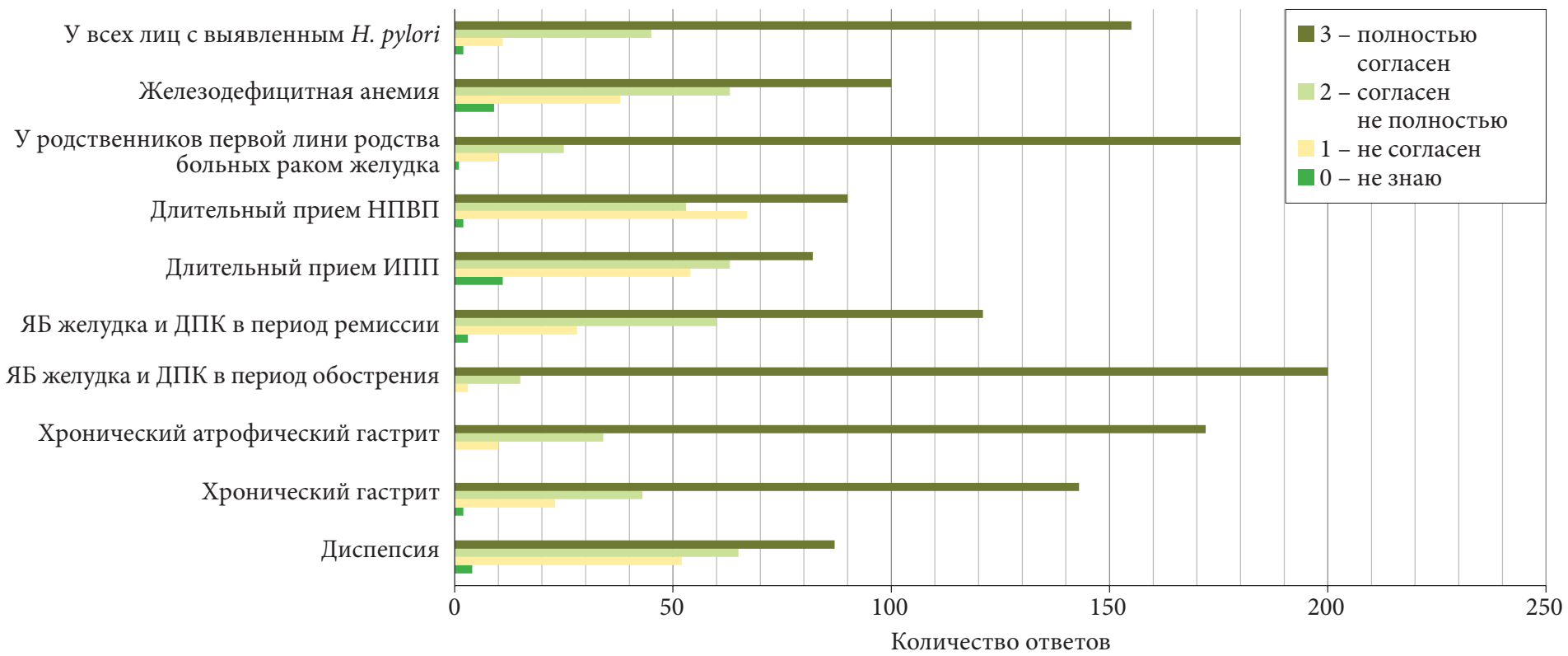

Рис. 1. Распределение врачей по степени согласия в отночении показаний к диагностике и лечению Н. pуlori

(НПВП - нестероидные противовоспалительные препараты, ИПП - ингибиторы протонной помпы, ЯБ - язвенная болезнь, ДПК - двенадцатиперстная кишка)

\section{Введение}

Международные и российские рекомендации по диагностике и лечению заболеваний, ассоциированных с инфекцией Helicobacter pylori, разработанные экспертами на основании данных рандомизированных клинических исследований, а также систематических обзоров и метаанализов, базирующихся на принципах доказательной медицины, демонстрируют высокую эффективность современных схем эрадикационной терапии [1-4]. Достоверность результатов всецело зависит от приверженности как пациента, так и врача соблюдению протокола исследования. Однако в практической деятельности мы сталкиваемся с рядом проблем, затрудняющих выполнение предписанных рекомендаций [5]. В связи с этим особую актуальность приобретают оценка приверженности врачей рекомендациям в регионах и разработка мер по устранению трудностей.

Цель работы - оценить приверженность врачей первичного звена выполнению рекомендаций по диагностике и лечению заболеваний, ассоциированных с H. pylori.

\section{Материал и методы}

Проведено анкетирование 221 врача, работающего в лечебно-профилактических учреждениях пяти городов европейской и центральной части России: Москвы (14\% числа опрошенных), Челябинска (1\%), Омска (44\%), Новосибирска (5\%), Кемерово (37\%). Сбор данных проводился на основании результатов добровольного анонимного анкетирования врачей с помощью опросника. Опросник включал девять вопросов, позволявших оценить знание и степень согласия специалистов с утверждениями, касающимися показаний к диагностике и лечению инфекции H. pylori, методов ее первичной диагностики и условий получения достоверных результатов исследований, наиболее эффективных схем антихеликобактерной терапии первой линии, методов повышения эффективности лечения, особенностей проведения эрадикационной терапии, методов и сроков проведения контроля эффективности эрадикации H. pylori, условий, затрудняющих диагностику инфекции и проведение эффективной эрадикации. Анкетирование во всех центрах предполагало использование единого опросника. Уровень согласия респонденты выражали в баллах: 0 не знаю, 1 - не согласен, 2 - согласен не полностью, 3 - полностью согласен. Обработка результатов исследования и графический анализ данных выполнены на персональном компьютере с помощью программ Microsoft Excel,
Statistica 6.1 методом статистической обработки медицинских данных [6]. Количественные данные представлены в виде среднего с указанием стандартного отклонения, качественные - в виде долей (\%) и абсолютных чисел. Для проверки нулевой гипотезы в номинальной шкале использован критерий сопряженности $\chi^{2}$ Пирсона. Сравнение двух не связанных друг с другом групп проводили с помощью критерия Манна - Уитни (Mann - Withney U test) для количественных данных. На всех этапах статистического анализа нулевая гипотеза отвергалась при $\mathrm{p} \leq 0,05$. Взаимосвязи между показателями оценивали с помощью корреляционного анализа Спирмена $\left(\mathrm{r}_{\mathrm{s}}\right)$.

\section{Результаты}

Среди респондентов насчитывалось $154(69,7 \%)$ терапевта и 67 (30,3\%) гастроэнтерологов. 157 $(71,4 \%)$ врачей работали в лечебно-профилактических учреждениях поликлинического типа, $46(20,8 \%)$ - в стационарах и 17 $(7,7 \%)$ - в частных центрах.

Возраст специалистов - от 22 до 81 года $(47,5 \pm 12,8$ года), стаж работы - от шести месяцев до 45 лет $(20,3 \pm 12,4$ года). Нами проведена оценка количества респондентов различных специальностей (тера- 




Рис. 2. Распределение врачей различных специальностей по степени согласия в отночении показаний к диагностике и лечению H. pylori (длительный прием ИПП). Сравнение методом $\chi^{2}$ Пирсона
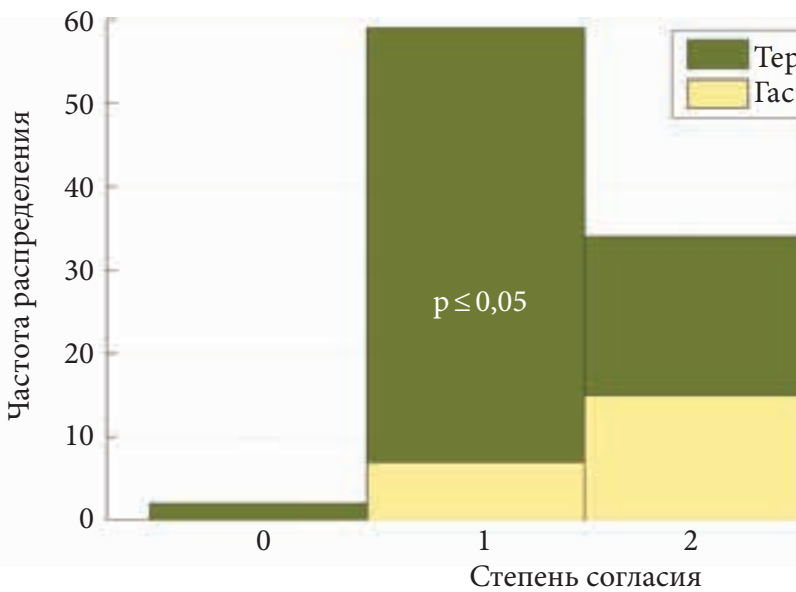

Рис. 3. Распределение врачей различных специальностей по степени согласия в отночении показаний к диагностике и лечению H. pylori (прием НПвП). Сравнение методом $\chi^{2}$ Пирсона певты и гастроэнтерологи) в зависимости от стажа (до десяти лет, 10-20 лет, 20-30 лет и свыше 30 лет) и квалификационной категории (ее отсутствие, первая, вторая и высшая). Результаты распределения врачей по стажу и квалификационной категории показали равные доли в группах терапевтов и гастроэнтерологов.

\section{Вопрос 1. Заболевания, при которых необходимо диагностировать и проводить эрадикацию инфекции H. pylori}

Результаты согласия в отношении показаний к диагностике и лечению H. pylori представлены на рис. 1. Лидирующие позиции по степени согласия у врачей всех специальностей заняли: язвенная болезнь в период обострения (90,5\%), родственники первой линии родства больных раком желудка $(81,4 \%)$, хронический атрофический гастрит $(77,8 \%)$. Врачей, которые бы не имели собственного мнения по данному вопросу, не было. Однако несогласие выразили до 10\%.

Интересно, что в отношении хронического атрофического гастрита достоверно чаще несогласными или не полностью согласными были именно терапевты $\left(\chi^{2}=14,6248 ; \mathrm{p} \leq 0,05\right)$ со стажем от десяти до 20 лет первой квалификационной категории $41(27,2 \%)$ терапевт против 2 (3,6\%) гастроэнтерологов.

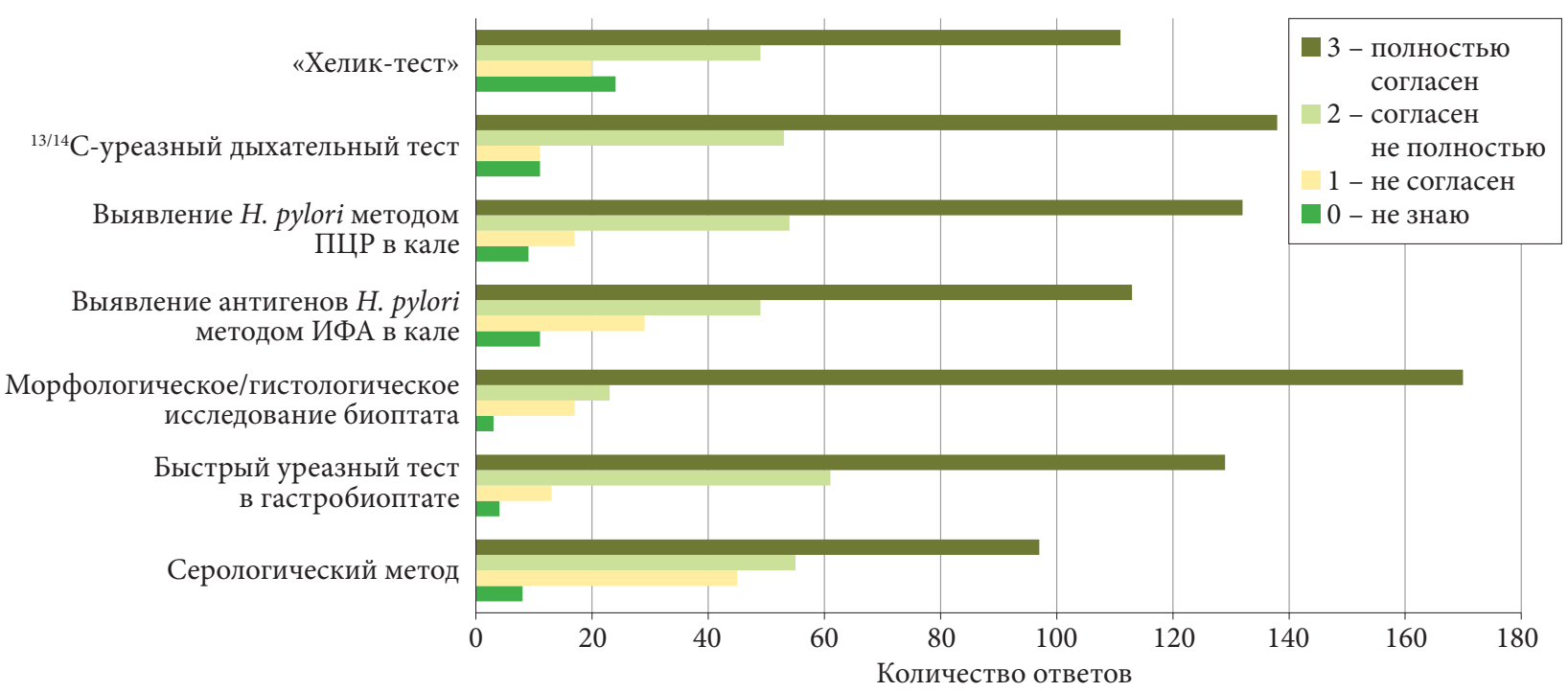

Примечание. ПЦР - полимеразная цепная реакция. ИФА - иммуноферментный анализ.

Рис. 4. Распределение врачей по степени согласия в отночении методов первичной диагностики H. pylori 
Практически равная степень согласия отмечалась для «хронического гастрита» $(64,7 \%)$ и «среди всех лиц, имеющих инфекцию $H$. pylori» $(70,1 \%)$. В отношении функциональной диспепсии согласие выразили $41,2 \%$ респондентов. При этом распределение ответов среди врачей различных специальностей, а также в зависимости от стажа и категории было одинаковым. Врачи были согласны диагностировать и проводить эрадикацию при планировании длительного приема ингибиторов протонной помпы (ИПП) - 37,1\%, нестероидных противовоспалительных препаратов (НПВП) - 40,7\% случаев. Несогласие достоверно чаще наблюдалось среди терапевтов со стажем до 20 лет, не имевших категории или первой квалификационной категории (для ИПП $\chi^{2}=15,2542 ; \mathrm{p} \leq 0,05$; для НПВП $\left.\chi^{2}=10,9537 ; \mathrm{p} \leq 0,05\right)$ (рис. 2 и 3). Обращает на себя внимание наличие тех, кто не сформировал собственного мнения, как среди терапевтов, так и среди гастроэнтерологов - в среднем до $10 \%$ числа опрошенных.

\section{Вопрос 2. Методы первичной диагностики инфекции H. pylori}

В качестве метода первичной диагностики $H$. pylori независимо от стажа, специальности и квалификационной категории респонденты назвали инвазивные методы (быстрый уреазный тест и/или гистобактериоскопию) $(76,9 \%)$ и серологический метод $(43,9 \%)$ (рис. 4). Гастроэнтерологи и терапевты одинаково часто были не согласны с использованием серологического метода (26,7 и $35,7 \%$ соответственно), а процент согласия был достоверно более высоким в группе терапевтов (51,4 против $\left.37,5 \%, \chi^{2}=9,1040 ; \mathrm{p} \leq 0,05\right)$.

Меньший процент использования дыхательных тестов и исследования кала с помощью иммуноферментного анализа (ИФА) и полимеразной цепной реакции (ПЦР) зарегистрирован среди терапевтов независимо от их стажа и категории. Достоверная значимость различия отмечалась в отношении «Хелик-теста» $\left(\chi^{2}=17,3588 ; \mathrm{p} \leq 0,05\right)$ (рис. 5).

Нами был предложен вариант ответа «Хелик-тест» ввиду сохраняющейся
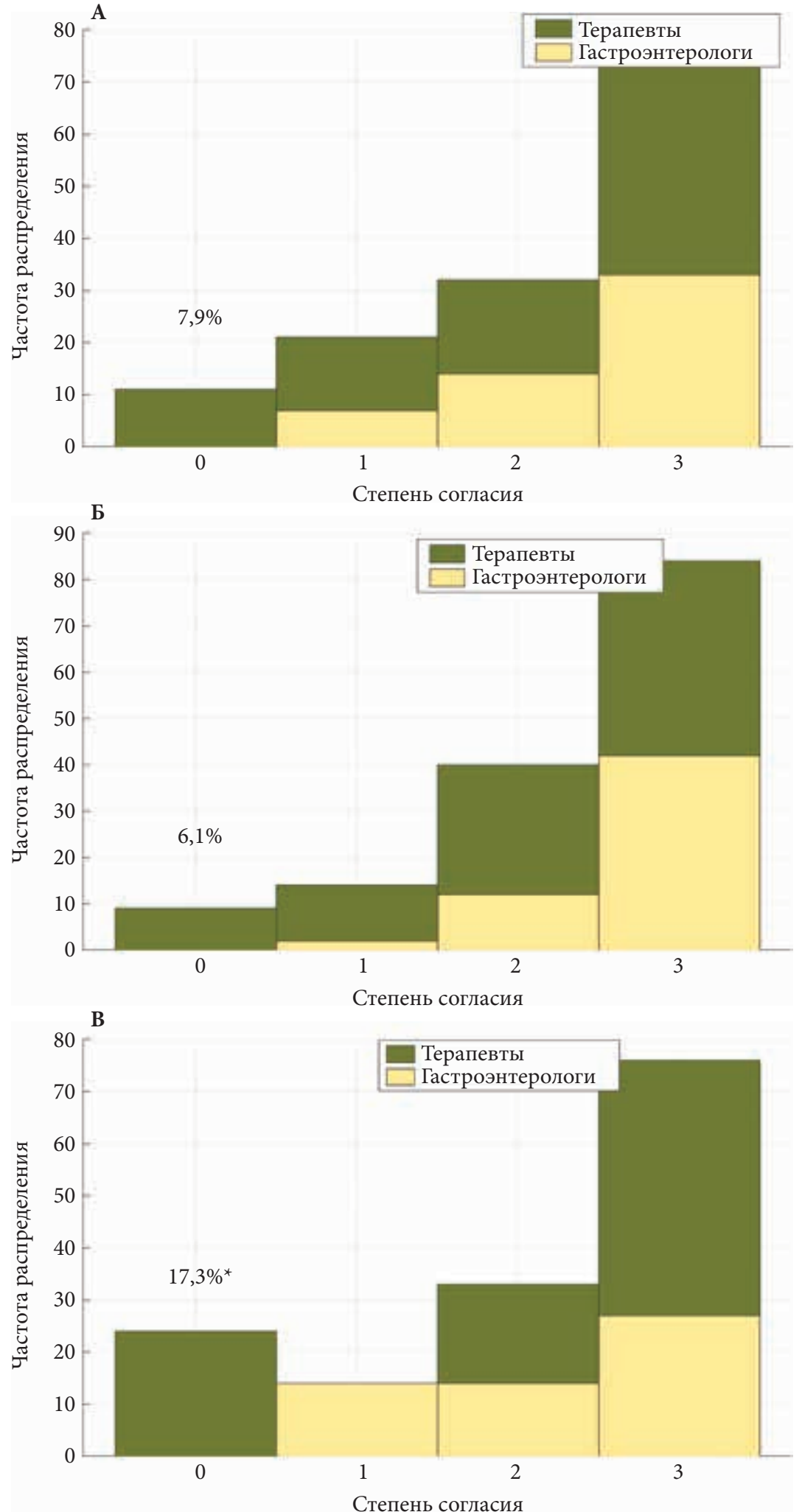

Рис. 5. Распределение врачей в зависимости от специальности по степени согласия в отношении определения антигенов Н. pylori методом ИФА в кале (A), ПЦР в кале (Б) и использования «Хелик-теста» (В) в качестве первичной диагностики H. pylori. Сравнение методом $\chi^{2}$

* Различия статистически значимы $(\mathrm{p} \leq 0,05)$. 
Отсутствие приема ИПП не менее чем за две недели до исследования

Отсутствие приема антибиотиков не менее чем за четыре недели до исследования

Отсутствие приема препарата висмута не менее чем за четыре недели до исследования

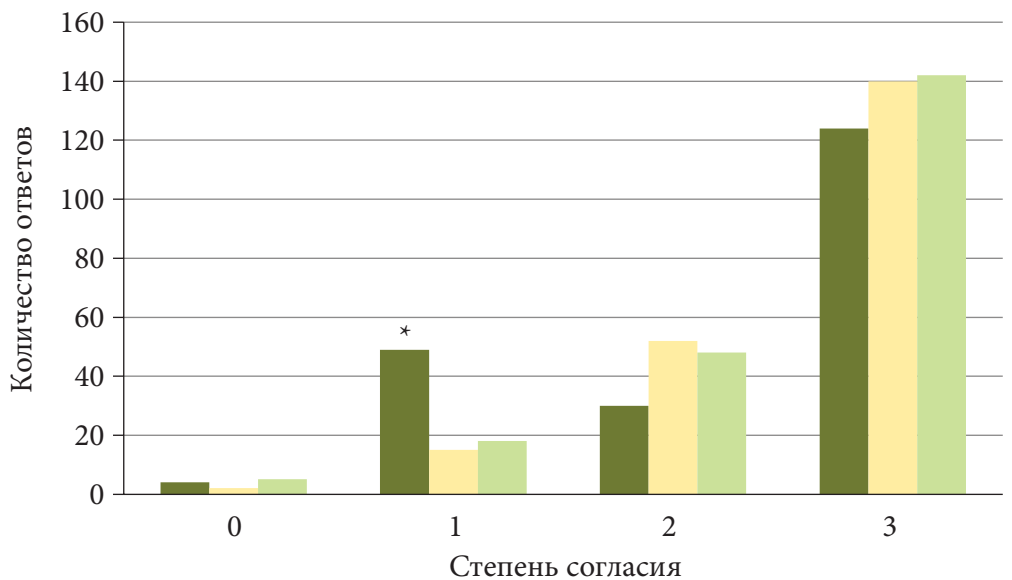

Рис. 6. Распределение респондентов по степени согласия в отночении методов получения достоверных результатов диагностики Н. pylori. Сравнение методом $\chi^{2}$ Пирсона

* Различия статистически значимы $(\mathrm{p} \leq 0,05)$.

Тройная терапия с джозамицином (ИПП + джозамицин + амоксициллин)

Последовательная терапия

Квадротерапия

(ИПП + тетрациклин + метронидазол + висмут $)$

Тройная терапия, усиленная висмутом (ИПП + кларитромицин + амоксициллин + висмут)

Стандартная тройная терапия (ИПП + кларитромицин + амоксициллин)

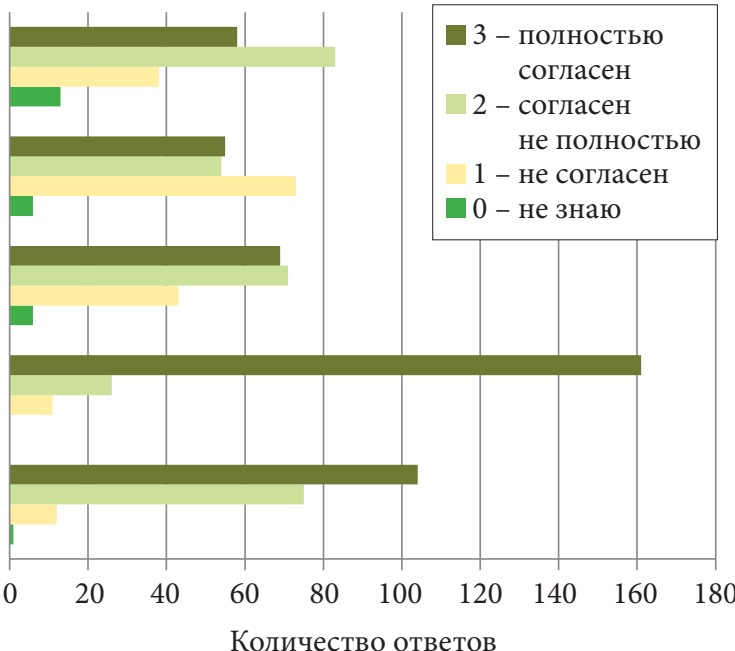

Рис. 7. Распределение респондентов по степени согласия в отночении выбора эрадикационной терапии первой линии

возможности применения этого теста в ряде российских регионов. Следует признать, что диагностические характеристики «Хелик-теста» подвергаются критике и его не рекомендуется применять для неинвазивной диагностики H. pylori [7].

\section{Вопрос 3. Что необходимо для получения достоверных результатов диагностики инфекции H. pylori?}

Терапевты и гастроэнтерологи независимо от стажа были согласны или практически согласны с необходимостью прекращения приема антибактериальных препаратов и препаратов висмута не менее чем за четыре недели до исследования
(86,9 и 85,97\%), ИПП не менее чем за две недели (69,7\%). Несогласие было достоверно выше среди терапевтов $\left(\chi^{2}=14,5196 ; p \leq 0,05\right)$ в отношении применения ИПП перед исследованием. Имела место достоверно значимая корреляция данного ответа в зависимости от стажа - от десяти до 30 лет и квалификационной категории - первой или второй (рис. 6).

\section{Вопрос 4. Какая, по вашему мнению, схема эрадикационной терапии первой линии наиболее эффективна?}

Среди схем лечения врачи чаще выбирали стандартную тройную терапию с кларитромицином $(80,9 \%)$, достоверно чаще терапевты $\left(\chi^{2}=18,2540 ; \mathrm{p} \leq 0,05\right)$. Тех, кто был согласен с добавлением к этой схеме терапии препаратов висмута, оказалось больше - 84,6\% (одинаковое число среди врачей разных специальностей) (рис. 7).

Мнение врачей в зависимости от специальности достоверно значимо отличалось в отношении квадротерапии с препаратами висмута и последовательной терапии $\left(\chi^{2}=7,2975\right.$; $\mathrm{p} \leq 0,05$ и $\chi^{2}=15,2110 ; \mathrm{p} \leq 0,05$ соответственно). И в том и в другом вопросе терапевты были более привержены назначению данных схем в качестве терапии первой линии. В то же время гастроэнтерологи либо не были согласны с ними, либо выражали сомнения (рис. 8 и 9).

\section{Вопрос 5. Особенности проведения эрадикационной терапии}

Наибольший процент полного согласия $(74,6 \%)$ отмечался в отношении 14-дневной эрадикационной терапии. С семидневной терапией согласилось менее $10 \%$ респондентов (все терапевты), с десятидневной - 24,8\%. В отношении коррекции дозы ИПП или их отмены у пациентов с хроническим атрофическим гастритом мнения разделились: согласных насчитывалось 23,1\% и несогласных - 24,4\%. Стаж, специальность или квалификация значения не имели. Большинство врачей $(52,5 \%)$ не согласились корректировать дозу препаратов - компонентов эрадикационной терапии в зависимости от веса пациента.

\section{Вопрос 6. Как можно повысить эффективность эрадикационной терапии?}

Врачам предложили следующие варианты ответов:

$\checkmark$ применение в качестве ИПП рабепразола или эзомепразола;

$\checkmark$ двойная доза ИПП;

$\checkmark$ добавление к терапии пробиотиков, препаратов висмута.

Распределение по степени согласия представлено на рис. 10. Большинство респондентов сочли необходимым добавление препаратов висмута. Достоверные различия среди врачей разных специальностей зафиксированы в отношении удвоения дозы ИПП $\left(\chi^{2}=12,4764 ; p \leq 0,05\right)$ : более приверженными оказались гастроэнтерологи. При этом несогласными как среди 
терапевтов, так и среди гастроэнтерологов были лица со стажем до 20 лет без квалификационной категории. Незнание по данному вопросу продемонстрировали только терапевты.

\section{Вопрос 7. Контроль эффективности после окончания терапии}

Согласие врачей на проведение контроля эрадикации зарегистрировано в 84,2\% случаев, причем не ранее чем через 30 дней по окончании лечения (90,1\%). 16 респондентов (8,6\% терапевтов и $5,8 \%$ гастроэнтерологов) не были согласны с проведением контрольных тестов. Количество пациентов, проходивших контроль после лечения у опрошенных врачей, в среднем составило 58,9 $\pm 29,4 \%$ с размахом от 10 до $100 \%$. Доля врачей, которые указали на выполнение контрольных тестов менее чем в 10\% случаев, составила 4,5\% (11 врачей). $91(41,2 \%)$ специалист затруднился ответить на данный вопрос.

\section{Вопрос 8. Методы контроля эрадикации по окончании терапии}

В качестве наиболее подходящего теста для контроля эрадикации врачи различных специальностей, как и в случае с первичной диагностикой, предпочли гистобактериоскопию 64,7\% случаев, быстрый уреазный тест - 29,4\%. Зафиксирован больший процент согласия с применением ${ }^{13 / 14} \mathrm{C}$-уреазного дыхательного теста $(58,4 \%)$ и ПЦР в кале $(55,7 \%)$ по сравнению с первичной диагностикой. Серологический метод готовы использовать 14,5\% специалистов, достоверно чаще терапевты $\left(\chi^{2}=31,5219 ; \mathrm{p} \leq 0,05\right)$.

\section{Вопрос 9. Что, по вашему мнению, затрудняет выполнение международных и российских рекомендаций по диагностике и лечению инфекции H. pylori?}

Респонденты в большей степени обеспокоены низкой приверженностью пациентов лечению $(93,4 \%)$ и ограниченностью в диагностических возможностях (85,3\%). На недостаточную эффективность и/или безопасность существующих схем лечения указали $40,8 \%$ респондентов. Дефицит времени на приеме отметили $85,3 \%$ врачей, достоверно чаще терапевты со стажем более 20 лет и второй и/или

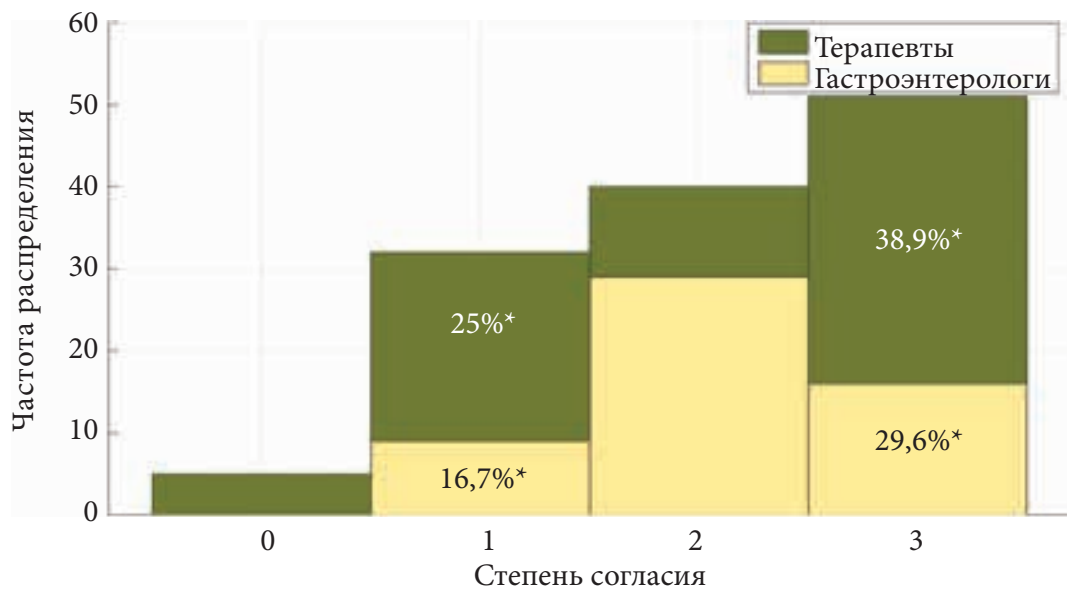

Примечание. Проценты указаны от общего количества опрошенных.

Рис. 8. Распределение врачей различных специальностей по степени согласия в назначении квадротерапии в качестве терапии первой линии. Сравнение методом $\chi^{2}$ Пирсона

* Различия статистически значимы ( $\mathrm{p} \leq 0,05)$.

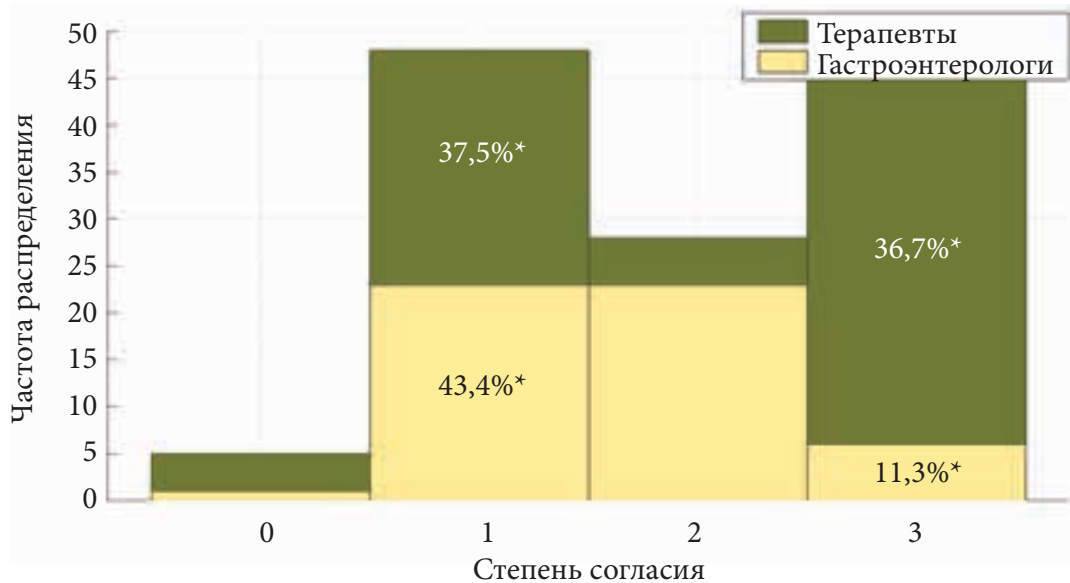

Примечание. Проценты указаны от общего количества опрошенных.

Рис. 9. Распределение врачей различных специиальностей по степени согласия в назначении последовательной терапии в качестве терапии первой линии. Сравнение методом $\chi^{2}$ Пирсона

* Различия статистически значимы $(\mathrm{p} \leq 0,05)$.

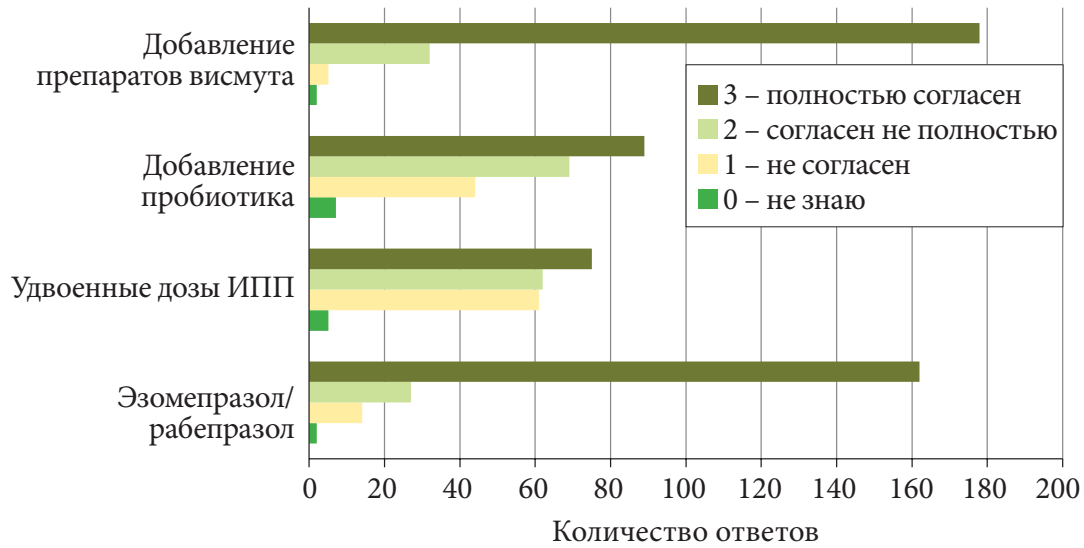

Рис. 10. Распределение респондентов по степени согласия с методами повышения эффективности эрадикационной терапии 
высшей квалификационной категорией $\left(\chi^{2}=18,2601 ; \mathrm{p} \leq 0,05\right)$.

\section{Обсуждение}

Согласно Киотскому соглашению, в основе классификации гастрита лежит этиологический принцип, а хронический гастрит, ассоциированный c H. pylori, рассматривается как инфекционное заболевание [1]. Данная инфекция ассоциирована с язвенной болезнью, атрофическим гастритом, аденокарциномой или MALT-лимфомой желудка $[8,9]$. Устранение H. pylori приводит к излечению гастрита, что является основой профилактики долгосрочных осложнений или рецидивов болезни. Не случайно в последних консенсусах отсутствует понятие абсолютных или относительных показаний к эрадикационной терапии. Она рекомендуется всем инфицированным взрослым пациентам в отсутствие противопоказаний [2]. В прежних согласительных документах в качестве обязательных для диагностики и устранения H. pylori рассматривались язвенная болезнь желудка и двенадцатиперстной кишки в стадии обострения и ремиссии, хронический гастрит, в том числе атрофический, наследственная предрасположенность к раку желудка, стадии раннего рака желудка, что не вызвало сомнения и у опрошенных нами врачей. Степень их согласия достигала 90,5\%, максимально при язвенной болезни в период обострения. Низкий процент согласия и высокий процент незнания зафиксированы в отношении таких показаний, как планируемый длительный прием ИПП, прием НПВП и неисследованная диспепсия. Подобные результаты отмечались и ранее. Так, по данным опроса 261 врача из 15 городов России, первичную диагностику H. pylori при язвенной болезни и хроническом гастрите подтвердили 221 (85\%) и 219 (84\%) опрошенных соответственно, а при НПВП-гастропатии - 84 (32\%), раке желудка - 80 $(30,7 \%)$ респондентов [10].

По данным испанского опроса 430 специалистов первичного звена, диагностику H. pylori чаще проводят при язвенной болезни (95\% респондентов) и существенно реже $(39,2 \%)$ при функциональной диспепсии [11].

Корейские ученые установили, что $65 \%$ врачей первичного звена считают необходимым проводить активную диагностику инфекции H. pylori у пациентов с функциональной диспепсией. Лишь 9\% рекомендовали контроль эрадикации впоследствии [12].

Панъевропейское исследование продемонстрировало различную убежденность врачей первичного звена в необходимости тестирования и лечения H. pylori-инфекции: Великобритания $47 \%$, Польша - 40\%, Греция - 32\% [13]. Из 188 немецких врачей первичного звена на активную диагностику и эрадикацию H. pylori при функциональной диспепсии указали 66\%, отягощенном наследственном анамнезе в отношении рака желудка - 55\%, до начала терапии НПВП/аспирином $19 \%$ опрошенных [14].

Врачи первичного звена, отвечая на вопрос о тактике ведения лиц, инфицированных H. pylori, принимающих НПВП, в 66\% случаев допускали ошибки (отмена НПВП/аспирина, замена на другой препарат, монотерапия ИПП, игнорирование необходимости проведения эрадикации H. pylori) [15].

К настоящему моменту получены убедительные данные, что эрадикация H. pylori способствует излечению хронического гастрита и ликвидации его морфологических проявлений независимо от продолжения антисекреторной терапии [16-19].

Как известно, у ряда больных H. pylori является причиной симптомов диспепсии [20].

Метаанализ 17 исследований с участием 3566 пациентов показал купирование симптомов диспепсии после антихеликобактерной терапии. При этом снижение относительного риска составило $10 \%$ [21].

Принципиально важно, что гастрит, ассоциированный с H. pylori, должен быть исключен для установления надежного диагноза функциональной диспепсии $[1,2]$. Использование аспирина и НПВП рассматривается в качестве обязательного показания к диагностике и эрадикации H. pylori, поскольку у пациентов, инфицированных H. pylori, повышен риск развития язвы и ее осложнений [22].

Представленные данные свидетельствуют о низкой осведомленности врачей, прежде всего терапевтов с небольшим стажем, о необходимости диагностики и лечения H. pylori при планировании длительного приема ИПП и НПВП, а также наличии симптомов диспепсии.

Результаты нашего опроса, равно как и ранее опубликованных исследований, показывают, что стратегия «тестируй и лечи» не получила распространения среди российских врачей $[10,23]$. В рекомендациях по ведению первичных пациентов с симптомами диспепсии, принятых на XIX Съезде Научного общества гастроэнтерологов России (Санкт-Петербург, май 2018 г.), сказано, что, несмотря на наметившуюся тенденцию к снижению заболеваемости раком желудка в России, уровни заболеваемости и смертности от злокачественных новообразований верхних отделов пищеварительного тракта остаются высокими. В связи с этим в России выполнение эзофагогастродуоденоскопии показано всем пациентам с синдромом диспепсии независимо от возраста [24].

Ряд неинвазивных $\left({ }^{13} \mathrm{C}\right.$-уреазный дыхательный тест, определение антигена H. pylori в кале методом ПЦР) и инвазивных (быстрый уреазный тест, морфологическое исследование) методик надежны в отсутствие приема антисекреторных препаратов в течение предыдущих двух недель до тестирования, антибиотиков и препаратов висмута в течение четырех недель до проведения теста [1]. По данным нашего исследования, об этом хорошо осведомлены терапевты и гастроэнтерологи. Поскольку не исключены вероятность длительного приема ИПП в условиях коморбидности и самостоятельный прием ИПП для купирования изжоги или диспепсических расстройств в силу удобства применения и низкой стоимости [25], серологический метод сохраняет актуальность для первичной диагностики H. pylori у пациентов, paнее не получавших эрадикационную терапию. Но его использование в качестве контрольного теста является распространенной ошибкой.

Результаты представленного опроса согласуются с другими данными в отношении высокой частоты применения стандартной тройной терапии с кларитромицином в качестве терапии первой линии в России [22]. Следует отметить, что эффективность данной схемы зависит от резистентности $H$. pylori к кларитромицину. Схема может назначаться лишь в регионах, где уровень резистентности не превышает 15\% 
[2]. К сожалению, данные по регионам Российской Федерации недостаточны и противоречивы. В среднем резистентность к кларитромицину оценивается на уровне 8,3\% [3]. По данным других авторов, она достигает 32,6\% [26].

Одним из способов повышения эффективности тройной терапии является добавление препарата висмута. Его синергизм с антибиотиками способствует преодолению резистентности к макролидам. Кроме того, висмут характеризуется цитопротективными свойствами $[27,28]$. Данный подход используется большим числом врачей. Продолжительность эрадикационной терапии должна составлять 14 дней, сокращение до десяти дней возможно при наличии локальных данных об эффективности. По этому поводу у врачей сомнений не было. Однако $10 \%$ опрошенных применяют семидневные схемы, что недопустимо.

В вопросе выбора ИПП нами был отмечен высокий процент согласия о преимуществах использования в эрадикационных схемах рабепразола и эзомепразола, что объясняется высокой биодоступностью препаратов независимо от генетического полиморфизма цитохрома Р450. Это неоднократно подтверждено результатами сравнительных исследований $[29,30]$. Вместе с тем доказательная база о преимуществах этих ИПП подвергается критике [31]. При исследовании полиморфизма СYP2C19*2 среди больных язвенной болезнью, ассоциированной с H. pylori, проживающих на территории Омской области, отмечалось преобладание быстрых $(71,4 \%)$ и промежуточных «метаболизаторов» (18,4\%). При введении в схему лечения омепразола в стандартных дозах у таких пациентов эффективность эрадикации H. pylori была ниже, чем у медленных «метаболизаторов» $(\mathrm{p}=0,021)[32]$.

Для повышения эффективности эрадикационной терапии применяют ИПП, метаболизм которых существенно не зависит от генетических особенностей лекарственного метаболизма препаратов этого класса [33]. В консенсусе Маастрихт V для повышения эффективности эрадикационной терапии рекомендованы двойные дозы ИПП и отмечены преимущества рабепразола и эзомепразола. При этом удвоение доз ИПП в схемах эрадикационной терапии не отражено в инструкци- ях к применению препаратов данной группы, что может трактоваться как «не по показаниям» [34].

По данным нашего исследования, врачи рекомендовали проведение контрольных тестов после эрадикационной терапии в $58,9 \pm 29,4 \%$ случаев, несмотря на высокий процент согласия врачей с необходимостью данного мероприятия (84,2\%). 41,2\% врачей не смогли ответить на данный вопрос.

Авторы крупного российского исследования распространенности H. pylori среди врачей (1154 врача из 14 регионов) отметили, что около $20 \%$ специалистов отказывались принять участие в тестировании, не желая знать свой H. pylori-статус. Около 50\% докторов указали в анкете, что не будут принимать антибиотики в случае обнаружения инфекции, $20 \%$ - будут принимать только пробиотики или препараты висмута. Только 30\% врачей были готовы использовать рекомендованные схемы. В конечном итоге из 619 инфицированных врачей курс антихеликобактерной терапии прошли только 117 (18,9\%) [35].

В другом исследовании распространенности H. pylori среди медицинских работников Москвы и Казани, по данным ${ }^{13} \mathrm{C}$-уреазного дыхательного теста, показано, что готовность пройти эрадикацию выразили лишь 61,4\% инфицированных [36].

Одна из серьезных проблем - соблюдение требований по срокам проведения контрольного теста. В ходе наблюдательного исследования «ПАРАД» установлено, что в $62,3 \%$ случаев эффективность лечения контролируют менее чем через четыре недели после окончания терапии. Это существенное отклонение от рекомендаций [37].

Среди причин, затрудняющих выполнение рекомендаций по диагностике и лечению инфекции H. pylori, по результатам нашего исследования, были названы ограничения в диагностических возможностях $(85,3 \%)$, что подчеркивают и другие исследователи $[5,10]$, и низкая приверженность пациентов лечению $(93,4 \%)$.

Принципиальное значение приверженности терапии показано в открытом когортном проспективном рандомизированном многоцентровом исследовании с участием 350 пациентов из Омска и Новосибирска [38]. Врачи - участковые терапевты, давшие согласие на участие в исследовании, прошли обучение по диагностике и лечению $H$. pylori-ассоциированных заболеваний, а также методам установления психологического контакта с пациентом и его мотивации к проведению назначенной терапии. После формирования исследовательской когорты на основании критериев включения/исключения и процедуры рандомизации всем больным назначили эрадикационную терапию первой линии. Пациенты основной группы, получившие подробные разъяснения о важности диагностики и лечения инфекции H. pylori, приняли не менее $90 \%$ назначенных врачом препаратов. 27 пациентов группы сравнения, в которой предусматривалось аналогичное лечение, но без дополнительных действий, мотивировавших к строгому соблюдению назначенного лечения, 27 (15,8\% числа лиц, завершивших исследование) пациентов сообщили о пропуске приема средств. Количество таблеток, принятых в ходе проводимого лечения, составило менее $70 \%$ назначенных врачом. В основной группе уровень достигнутой эрадикации составил $86 \%$, в группе сравнения - 74,9\% числа лиц, завершивших лечение по протоколу $(\mathrm{p}<0,02)$. Отметим, что низкая приверженность антимикробной терапии ведет к накоплению в популяции резистентных возбудителей.

Таким образом, информированность пациента о сути заболевания, повышение его мотивации к соблюдению рекомендаций врача являются одной из составляющих успешного лечения $[39,40]$.

\section{Заключение}

Проведенный опрос выявил недостаточный уровень знаний врачей современных клинических рекомендаций по диагностике и лечению заболеваний, ассоциированных с H. pylori. Coблюдение рекомендаций зависит не только от уровня образования врача, доступности методов диагностики и лечения, но и готовности врача следовать им. На приверженность врача существенное влияние оказывают как осведомленность, так и недостаточная степень согласия. Эти данные необходимо учитывать при планировании образовательных мероприятий.

Авторы заявляют об отсутствии конфликта интересов. 


\section{Литература}

1. Sugano K., Tack J., Kuipers E.J. et al. Kyoto global consensus report on Helicobacter pylori gastritis // Gut. 2015. Vol. 64. № 9. P. 1353-1367.

2. Malfertheiner P., Megraud F., O'Morain C.A. et al. Management of Helicobacter pylori infection - the Maastricht V/Florence Consensus Report // Gut. 2017. Vol. 66. № 1. P. 6-30.

3. Ивашкин В.Т., Маев И.В., Лапина Т.Л. и др. Клинические рекомендации Российской гастроэнтерологической ассоциации по диагностике и лечению инфекции Helicobacter pylori у взрослых // Российский журнал гастроэнтерологии, гепатологии, колопроктологии. 2018. Т. 28. № 1. С. 55-70.

4. Chey W.D., Leontiadis G.I., Howden C.W., Moss S.F. ACG clinical guideline: treatment of Helicobacter pylori infection // Am. J. Gastroenterol. 2017. Vol. 112. № 2. P. 212-239.

5. Cheng H.C., Liou J.M., Luo J.C. et al. The implementation of the consensus on the management of Helicobacter pylori and barriers to consensus // Helicobacter. 2018. Vol. 23. № 5. P. e12533.

6. Глани С. Медико-биологическая статистика / пер. с англ. Ю.А. Данилова. М.: Практика, 1998.

7. Бордин Д.С., Войнован И.Н., Колбасников С.В., Эмбутниекс Ю.В. Методы диагностики инфекции Helicobacter pylori в клинической практике // Терапевтический архив. 2018. Т. 90. № 12. С. 133-139.

8. Мозговой С.И., Костенко М.Б., Кролевеи Т.С., Ливзан М.А. Аутоиммунный гастрит в фокусе клинициста и морфолога // Фарматека. 2019. Т. 26. № 2. С. 121-129.

9. Лазебник Л.Б., Ткаченко Е.И., Абдулганиева Д.И. и др. VI Национальные рекомендации по диагностике и лечению кислотозависимых и ассоциированных с Helicobacter pylori заболеваний (VI Московские соглашения) // Экспериментальная и клиническая гастроэнтерология. 2017. № 2 (138). С. 3-21.

10. Дехнич Н.Н., Козлов Р.С., Саблин О.А., Прищепова Е.А. Диагностика Helicobacter pylori и выбор эрадикационной терапии: результаты анкетирования врачей в различных регионах Российской Федерации // Российский журнал гастроэнтерологии, гепатологии, колопроктологии. 2018. T. 28. № 2. C. 33-41.

11. Cano-Contreras A.D., Rascón O., Amieva-Balmori M. et al. Approach, attitudes, and knowledge of general practitioners in relation to Helicobacter pylori is inadequate. There is much room for improvement! // Rev. Gastroenterol. Mex. 2018. Vol. 83. № 1. P. 16-24.

12. Kim B.G., Kim J.W., Jeong J.B. et al. Discrepancies between primary physician practice and treatment guidelines for $\mathrm{He}$ licobacter pylori infection in Korea // World J. Gastroenterol. 2006. Vol. 12. № 1. P. 66-69.

13. Seifert B., Rubin G., de Wit N. et al. The management of common gastrointestinal disorders in general practice A survey by the European Society for Primary Care Gastroenterology (ESPCG) in six European countries // Dig. Liver Dis. 2008. Vol. 40. № 8. P. 659-666.

14. Boltin D., Kimchi N., Dickman R. et al. Attitudes and practice related to Helicobacter pylori infection among primary care physicians // Eur. J. Gastroenterol. Hepatol. 2016. Vol. 28. № 9. P. 1035-1040.
15. Zullo A., Hassan C., Oliveti D. et al. Helicobacter pylori management in non-steroidal anti-inammatory drug therapy patients in primary care // Intern. Emerg. Med. 2012. Vol. 7. № 4. P. 331-335.

16. Kuipers E.J., Uyterlinde A.M., Peña A.S. et al. Increase of Helicobacter pylori-associated corpus gastritis during acid suppressive therapy: implications for longterm safety // Am. J. Gastroenterol. 1995. Vol. 90. № 3. P. 1401-1406.

17. Schenk B.E., Kuipers E.J., Nelis G.F. et al. Effect of Helicobacter pylori eradication on chronic gastritis during omeprazole therapy // Gut. 2000. Vol. 46. № 5. P. 615-621.

18. Kuipers E.J., Nelis G.F., Klinkenberg-Knol E.C. et al. Cure of Helicobacter pylori infection in patients with reflux oesophagitis treated with long term omeprazole reverses gastritis without exacerbation of reflux disease: results of a randomised controlled trial // Gut. 2004. Vol. 53. № 1. P. 12-20.

19. Ивашкин В.Т., Маев И.В., Абдулхаков Р.А. и др. Инфекция Helicobacter pylori при длительной терапии кислотозависимых заболеваний ингибиторами протонной помпы // Российский журнал гастроэнтерологии, гепатологии, колопроктологии. 2018. Т. 28. № 3. С. 26-32.

20. Drossman D.A., Hasler W.L. Rome IV - Functional GI disorders: disorders of gut-brain interaction // Gastroenterology. 2016. Vol. 150. № 6. P. 1257-1261.

21. Moayyedi P., Soo S., Deeks J. et al. Eradication of Helicobacter pylori for non-ulcer dyspepsia // Cochrane Database Syst. Rev. 2006. Vol. 2. CD 002096.

22. Насонов Е.Л., Ивашкин В.Т., Яхно Н.Н. и др. Проект Национальных клинических рекомендаций (основные положения) Ассоциации ревматологов России, Российской гастроэнтерологической ассоциации, Российского общества по изучению боли «Рациональное использование нестероидных противовоспалительных препаратов» // Российский журнал гастроэнтерологии, гепатологии, колопроктологии. 2017. Т. 27. № 5. С. 69-75.

23. Бордин Д.С., Эмбутниекс Ю.В., Вологжанина Л.Г. и др. Европейский регистр Helicobacter pylori (HP-EUREG): как изменилась клиническая практика в России с 2013 по 2018 год // Терапевтический архив. 2019. Т. 91. № 2. С. 16-24.

24. Лазебник Л.Б., Алексеенко С.А., Лялюкова Е.А. и др. Рекомендации по ведению первичных пациентов с симптомами диспепсии // Экспериментальная и клиническая гастроэнтерология. 2018. № 5 (153). С. 4-18.

25. Helicobacter Pylori in Developing Countries // World Gastroenterology Organisation Global Guidelines. 2010 //www. worldgastroenterology.org/UserFiles/file/guidelines/helicobacter-pylori-english-2010.pdf.

26. Цуканов В.В., Каспаров Э.В., Васютин А.В., Тонких Ю.Л. Эрадикация Helicobacter pylori // Медицинский совет. 2015. № 13. C. 26-28.

27. He X., Liao X., Li H. et al. Bismuth-induced inactivation of ferric uptake regulator from Helicobacter pylori // Inorg. Chem. 2017. Vol. 56. № 24. P. 15041-15048.

28. Tulassay Z., Herszenyi L. Gastric mucosal defense and cytoprotection // Best Pract. Res. Clin. Gastroenterol. 2010. Vol. 24. № 2. P. 99-108.

29. McNicholl A.G., Linares P.M., Nyssen O.P. Metaanalysis: esomeprazole or rabeprazole vs. first-generation pump inhibitors in the treatment of Helicobacter pylori infection // Aliment. Pharmacol. Ther. 2012. Vol. 36. № 5. P. 414-425. 
30. Kirchheiner J., Glatt S., Fuhr U. et al. Relative potency of proton-pump inhibitors - comparison of effects on intragastric pH // Eur. J. Clin. Pharmacol. 2009. Vol. 65. № 1. P. 19-31.

31. Никонов Е.Л., Солодовников А.Г., Кайбышева В.О. и др. Критический анализ публикации «Метаанализ: эзомепразол или рабепразол в сравнении с ингибиторами протонной помпы первого поколения в лечении инфекции» // Доказательная гастроэнтерология. 2018. Т. 7. № 4. С. 44-64.

32. Елохина Е.В., Костенко М.Б., Ливзан М.А., Скальский С.В. Эффективность эрадикационной терапии у пациентов с язвенной болезнью, ассоциированной с Helicobacter pylori, в зависимости от генотипа лекарственного метаболизма ингибиторов протонной помпы // Экспериментальная и клиническая гастроэнтерология. 2015. № 3 (115). C. 31-33.

33. Tang H.L., Li Y., Hu Y.F. et al. Effects of CYP2C19 loss-offunction variants on the eradication of $\mathrm{H}$. pylori infection in patients treated with proton pump inhibitor based triple therapy regimens: a meta-analysis of randomized clinical trials // PLoS One. 2013. Vol. 8. № 4. P. e62162.

34. Андреев Д.Н., Дичева Д.Т., Маев И.В. Возможности оптимизации эрадикационной терапии инфекции Helicobacter pylori в современной клинической практике // Терапевтический архив. 2017. Т. 89. № 2. С. 84-90.
35. Бакулина Н.В., Симаненков В.И., Бакулин И.Г., Ильчищина Т.А. Распространенность хеликобактерной инфекции среди врачей // Экспериментальная и клиническая гастроэнтерология. 2017. № 12 (148). С. 20-24.

36. Бордин Д.С., Плавник Р.Г., Невмержицкий В.И. и др. Распространенность Helicobacter pylori среди медицинских работников Москвы и Казани по данным ${ }^{13} \mathrm{C}$-уреазного дыхательного теста // Альманах клинической медицины. 2018. T. 46. № 1. С. 40-49.

37. Лазебник Л.Б., Бордин Д.С. Диагностика и лечение заболеваний, ассоциированных с Helicobacter pylori, в условиях реальной клинической практики: результаты наблюдательной программы «ПАРАД» // Вестник практического врача. 2014. Т. 3. № 3. С. 31-42.

38. Осипенко М.Ф., Ливзан М.А., Бикбулатова Е.А. «Комплаентность» пациента как один из факторов, определяющих эффективность эрадикационной терапии // Терапевтический архив. 2014. Т. 86. № 2. С. 27-31.

39. Бордин Д., Белоусова Н., Воробъева Н., Зеленикин С. Язвенная болезнь: пути повышения эффективности эрадикации Helicobacter pylori // Врач. 2011. № 4. С. 43-47.

40. Wang C.H., Liao S.T., Yang J. et al. Effects of daily telephonebased re-education before taking medicine on Helicobacter pylori eradication: a prospective single-center study from China // World J. Gastroenterol. 2015. Vol. 21. № 39. P. 11179-11184.

\title{
Consent of Doctors to Guidelines for the Diagnosis and Treatment of Diseases Associated with Helicobacter pylori
}

D.S. Bordin, $\mathrm{MD}$, PhD, Prof. ${ }^{1-3}$, T.S. Krolevets, $\mathrm{PhD}^{4}$, M.A. Livzan, MD, PhD, Prof. , M.F. Osipenko, MD, PhD, Prof. ${ }^{5}$, L.I. Tokareva ${ }^{6}$, Ye.V. Chebanenko ${ }^{4}$

\author{
${ }^{1}$ A.S. Loginov Moscow Clinical Scientific and Practical Center \\ ${ }^{2}$ Tver State Medical University \\ ${ }^{3}$ A.I. Yevdokimov Moscow State University of Medicine and Dentistry \\ ${ }^{4}$ Omsk State Medical University \\ ${ }^{5}$ Novosibirsk State Medical University \\ ${ }^{6}$ Kemerovo Clinical Hospital
}

Contact person: Tatyana S. Krolevets, mts-8-90@mail.ru

Purpose - to assess the commitment of doctors to follow the recommendations for the diagnosis and treatment of $\mathrm{H}$. pylori-associated diseases.

Material and methods. It was conducted an anonymous survey among 211 internists ( $n=154 ; 69.7 \%$ ) and gastroenterologists $(n=57 ; 25.8 \%)$, working in outpatient departments in different cities of the European and central part of Russia. The level of agreement was expressed by the degree: 0 - I don't know, 1 - disagree, 2 - not fully agree and 3 - fully agree.

Results. The doctors agreed to diagnose and eradicate HP in case of peptic ulcer in exacerbation (90.5\%), in close relatives with stomach cancer (81.4\%), and in case of chronic atrophic gastritis (77.8\%), as well as in long-term intake of PPI in $38.9 \%$ cases, and NSAID in $42.6 \%$ cases. Disagreement was significantly essential among therapists with experience up to 20 years without any categories $\left(\chi^{2}=10.9537 ; p \leq 0.05 ; \chi^{2}=15.2542 ; p \leq 0.05\right)$. Doctors preferred using histobacterioscopy of gastrobioptat for diagnosis of HP both initially and as a control test after treatment (76.9 and 64.7\%) in comparison with serological methods (43.9 and 14.5\%). Doctors were less willing to use the respiratory test and stool test, both initially (56.3 and 55.4\%) and after treatment (58.4 and 55.7\%). Among treatment regimens, doctors follow the standard triple therapy with Kla (80.9\%), it was significantly higher among therapists $\left(\chi^{2}=18.2540 ; p \leq 0.05\right)$. Among the reasons for non-compliance with the recommendations the respondents noted low persistence of patients (93.4\%) and limited diagnostic facilities (85.3\%).

Conclusion. Inadequate agreement of therapists to recommendations may underlie the inefficiency of $H$. pylori diagnosis and treatment.

Key words: commitment, diagnosis and treatment of Helicobacter pylori, recommendations 